intervention $=70$ ). Wellbeing was measured with the Hospital Anxiety and Depression Scale (HADS) and Impact Event Scale (IES).

Results No significant differences were found in wellbeing of FCs between groups. The mean scores for the HADS show no significant differences between groups for anxiety (mean score control 7,09 vs. mean score intervention 8,29) and depression (mean score control 6,72 vs. mean score intervention 7,17). No significant differences are found between groups in the mean scores for the IES. Intrusion had a mean score of 21,27 for control vs. 21,38 for intervention; Avoidance had a mean score of 10,34 for control vs. 12,72 for intervention.

Conclusion Despite previous evidence about improved outcomes for wellbeing in FCs in ACP programs, our ACP intervention did not show differences between groups. Possibly the non-response or cultural discrepancies between the different countries have a part in this. More research is needed to explain what mechanisms are present.

This study is supported by a grant from the FWO (nr. G034717N).

\section{OP50 INFLUENCING FACTORS ON ENGAGEMENT IN ADVANCE CARE PLANNING (ACP) FROM THE CAREGIVER'S PERSPECTIVE}

S Herzog, M Koch, C Seifart. Philipps-University Marburg, Marburg, Germany

\subsection{6/spcare-2019-ACPICONGRESSABS.50}

Background One important aspect of successful ACP is the engagement of caregivers during the process. However, sometimes the engagement of patients and their caregivers in ACP is known to be difficult. Therefore barriers and facilitators of participation in ACP from the caregiver's point of view are explored.

Methods 12 relatives (caregivers) of palliative-oncologic patients were questioned in guideline-based interviews. 5 caregivers were children and 7 were spouses of patients. The analysis was conducted by Qualitative Content Analysis by $\mathrm{Ma}$ Recruiting patients yring. Two code trees were built: one posing a two-dimensional axis system made of plus and minus poles, the other one operating in the shape of clusters ("clouds") considering overlappings and connections of the six main themes ("stardust model").

Results Six result clusters were evaluated influencing the willingness of both patients and caregivers.

1. Skills Of ACP-Initiators (general and social expectations on the initiator and conductor of ACP-intervention)

2. "Omnipresent Electivity" (setting of daily-life impulses for ACP bearing a non-binding nature)

3. Importance of Relationship Between Spouses (understanding of symmetrical needs)

4. Relative Differences Of Perception Of Children And Spouses of patients

5. Maintenance of Objective Necessities (non-emotional structural circumstances such as resources of time, finances and legal responsibilities)

All empowering relatives to be

6. A Participative Caregiver (including experiences and desired role in ACP setting)

Conclusions Acknowledging the importance of caregivers and their essential role in ACP further interventions should consider incorporating these factors to improve the implementation of such.

\section{OP51 EXPERIENCES WITH AND OUTCOMES OF ADVANCE CARE PLANNING IN BEREAVED RELATIVES OF FRAIL OLDER PATIENTS: A MIXED METHODS STUDY}

${ }^{1}$ A Overbeek*, 'I Korfage, ${ }^{2} \mathrm{~B}$ Hammes, ${ }^{1} \mathrm{~A}$ van der Heide, 'J Rietjens. ${ }^{1}$ Erasmus MC, Rotterdam, Netherlands; ${ }^{2}$ C-TAC Innovations, La Crosse, USA

\subsection{6/spcare-2019-ACPICONGRESSABS.51}

Background Advance Care Planning (ACP) may prepare relatives of frail older patients for future decision-making.

Objective To investigate (1) how bereaved relatives of frail older patients experience ACP conversations and (2) whether ACP has an effect on relatives' preparation for decision-making and on their levels of anxiety and depression.

Design: Cluster randomised controlled trial.

Setting: Residential care homes in the Netherlands.

Subjects: Bereaved relatives of care home residents and community-dwelling frail older patients.

Methods We randomised 16 residential care homes to either the intervention group, where participants (frail older patients) were offered facilitated ACP, or the control group $(n=201)$, where they received 'care as usual'. If participants died, we approached relatives for an interview. We asked relatives who had attended ACP conversations for their experience with ACP (open-ended questions). Furthermore, we compared relatives' preparation levels for decision-making and levels of anxiety and depression (HADS) between groups. This trial was registered (NTR4454).

Results We conducted interviews with 39/51 (76\%) bereaved relatives (intervention group: $n=20$, control group: $n=19$ ). Relatives appreciated the ACP conversations. A few considered ACP redundant since they were already aware of the patient's preferences. Nine of 10 relatives in the intervention group felt adequately prepared for decision-making as compared to five of 11 relatives in the control group $(p=.03)$. Relatives' levels of anxiety and depression did not differ significantly between groups.

Conclusions In our study, bereaved relatives of frail older patients appreciated ACP. ACP positively affected preparedness for decision-making. It did not significantly affect levels of anxiety or depression.

\section{OP52 TO WHAT EXTENT DO ONLINE RESOURCES MEET THE NEEDS OF SUBSTITUTE DECISION-MAKERS IN AUSTRALIA? PART 2}

${ }^{1} \mathrm{M}$ Sellars*, ${ }^{1} \mathrm{~J}$ Tran, ${ }^{1} \mathrm{~L}$ Nolte, ${ }^{2} \mathrm{~B}$ White, ${ }^{3} \mathrm{C}$ Sinclair, ${ }^{4} \mathrm{D}$ Fetherstonhaugh, ${ }^{5} \mathrm{~K}$ Detering. ${ }^{7}$ Austin Health, MELBOURNE, Australia; ${ }^{2}$ Australian Centre of Health Law Research, Queensland University of Technology, Queensland, Australia; ${ }^{3}$ Rural Clinical School of Western Australia, University of Western Australia, Albany, Western Australia, Australia; ${ }^{4}$ Australian Centre for Evidence Based Aged Care, La Trobe University, Melbourne, Victoria, Australia; ${ }^{5}$ Faculty of Medicine, Dentistry and Health Science, University of Melbourne, Melbourne, Victoria, Australia

\subsection{6/spcare-2019-ACPICONGRESSABS.52}

Background We aimed to examine Australians' knowledge, attitudes and experiences regarding the role of substitute 